\title{
Efficient Syntheses of the Keto-carotenoids - Canthaxanthin, Astaxanthin, and Astacene
}

\author{
Seyoung Choi and Sangho Koo* \\ Department of Chemistry, Myong Ji University, Yongin, Kyunggi-Do, 449-728, Korea. \\ E-mail: sangkoo@mju.ac.kr
}

Table of Contents

Experimental Section----------------------------------------------------------S2

${ }^{1}$ H NMR Spectrum of Compound 1----------------------------------------S11

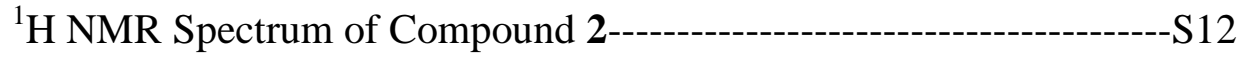

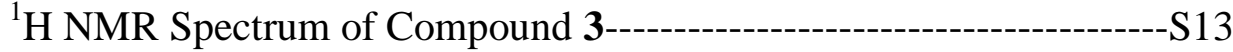

${ }^{1}$ H NMR Spectrum of Compound 7----------------------------------------S14

${ }^{1}$ H NMR Spectrum of Compound 8-----------------------------------------S15

${ }^{1} \mathrm{H}$ NMR Spectrum of Compound 10b---------------------------------------S16

${ }^{1} \mathrm{H}$ NMR Spectrum of Compound 10c--------------------------------------S17

${ }^{1}$ H NMR Spectrum of Compound 10d--------------------------------------S18

${ }^{1} \mathrm{H}$ NMR Spectrum of Compound 10e-------------------------------------S19

${ }^{1}$ H NMR Spectrum of Compound 11b---------------------------------------S20

${ }^{1} \mathrm{H}$ NMR Spectrum of Compound 11c---------------------------------------S21

${ }^{1} \mathrm{H}$ NMR Spectrum of Compound 11e----------------------------------------S22 


\section{Experimental Section}

General Experimental. ${ }^{1} \mathrm{H}(300 \mathrm{MHz})$ and ${ }^{13} \mathrm{C}$ NMR $(75.5 \mathrm{MHz})$ spectra were recorded in $\mathrm{CDCl}_{3}$ with $\mathrm{Me}_{4} \mathrm{Si}(\delta=0 \mathrm{ppm})$ as an internal standard. Solvents for extraction and chromatography were reagent grade and used as received. Column chromatography was performed by the method of Still using silica gel 60, 230-400 mesh ASTM supplied by Merck. Solvents used as reaction media were dried over pre-dried molecular sieve $(4 \AA$ ) by microwave oven. All reactions were performed under dry argon in oven-dried glassware, except for those reactions with $\mathrm{H}_{2} \mathrm{O}$ as a solvent, which were run in air.

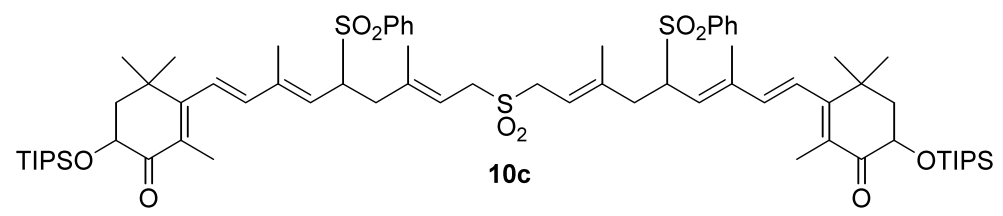

Bis(5-benzenesulfonyl-3,7-dimethyl-9-(3-oxo-4-triisopropylsiloxy-2,6,6-trimethyl-1-cyclohexenyl)-

2,6,8-nonatrienyl) sulfone (10c). Following the general procedure for 10b, keto-trisulfone 7 (3.82 g, 4.17 mmol) reacted with triisopropylsilyl trifluoromethanesulfonate $(2.50 \mathrm{~mL}, 9.17 \mathrm{mmol})$ in $\mathrm{CH}_{2} \mathrm{Cl}_{2}(40 \mathrm{~mL})$ under $\mathrm{Et}_{3} \mathrm{~N}(3.00 \mathrm{~mL}, 20.85 \mathrm{mmol})$ at room temperature for $2 \mathrm{~h}$ to give $9 \mathrm{c}(6.71 \mathrm{~g})$ as a white solid.

The oxidation of $9 \mathbf{c}(6.71 \mathrm{~g})$ with perphthalic acid, which was prepared by the reaction of urea- $\mathrm{H}_{2} \mathrm{O}_{2}$ (UHP, $3.92 \mathrm{~g}, 41.70 \mathrm{mmol})$ and phthalic anhydride $(3.09 \mathrm{~g}, 20.85 \mathrm{mmol})$ in $\mathrm{CH}_{3} \mathrm{CN}(40 \mathrm{~mL})$, under $\mathrm{Na}_{2} \mathrm{CO}_{3}(4.42 \mathrm{~g}$, $41.70 \mathrm{mmol})$ in $\mathrm{CH}_{2} \mathrm{Cl}_{2}(40 \mathrm{~mL})$ at room temperature for $10 \mathrm{~h}$ gave crude $10 \mathrm{c}$ as a light-yellow solid $(4.52 \mathrm{~g}$, $3.59 \mathrm{mmol}, 86 \%)$. The crude product was purified by flash column chromatography on silica gel which was deactivated by $\mathrm{Et}_{3} \mathrm{~N}$ to give $10 \mathrm{c}(3.78 \mathrm{~g}, 3.00 \mathrm{mmol})$ in $72 \%$ yield, which was consisted of $\sim 1: 1$ diastereoisomers presumably at the carbons containing the etheral group. 
Data for 10c: ${ }^{1}$ H NMR $\delta$ 1.05-1.18 (m, 48H), 1.20 (s, 6H), ${ }^{*} 1.24$ (s, 6H), 1.32 (s, 6H), $1.34(\mathrm{~s}, 6 \mathrm{H}),{ }^{*} 1.69$ (s, 6H), $1.77(\mathrm{~s}, 6 \mathrm{H}),{ }^{*} 1.78(\mathrm{~s}, 6 \mathrm{H}), 1.99(\mathrm{~d}, J=8.3 \mathrm{~Hz}, 4 \mathrm{H}), 2.48(\mathrm{dd}, J=12.6,11.9 \mathrm{~Hz}, 2 \mathrm{H}), 3.04(\mathrm{~d}, J=$ $14.6 \mathrm{~Hz}, 2 \mathrm{H}), 3.40-3.63(\mathrm{~m}, 4 \mathrm{H}), 4.09$ (br t, $J=9.6 \mathrm{~Hz}, 2 \mathrm{H}), 4.43(\mathrm{dd}, J=8.8,2.2 \mathrm{~Hz}, 2 \mathrm{H}), 4.46$ (dd, $J=7.7$, $2.3 \mathrm{~Hz}, 2 \mathrm{H}),{ }^{*} 5.23(\mathrm{~d}, J=10.8 \mathrm{~Hz}, 2 \mathrm{H}), 5.28(\mathrm{br} \mathrm{s}, 2 \mathrm{H}), 6.04\left(\mathrm{~d}\right.$ of A of ABq, $\left.J_{A B}=16.4, J_{d}=1.7 \mathrm{~Hz}, 2 \mathrm{H}\right)$, $6.10\left(\mathrm{~d}\right.$ of B of ABq, $\left.J_{A B}=16.4, J_{d}=2.1 \mathrm{~Hz}, 2 \mathrm{H}\right), 7.44-7.56(\mathrm{~m}, 4 \mathrm{H}), 7.61-7.68(\mathrm{~m}, 2 \mathrm{H}), 7.75-7.84(\mathrm{~m}, 4 \mathrm{H})$ ppm; ${ }^{13} \mathbf{C}$ NMR $\delta 12.3,12.4,13.9,17.0,17.9,18.0,26.2,30.2,30.3, * 36.8,37.5,47.1,51.3,63.4,70.8,113.8$, $124.6,{ }^{*} 124.7,126.2,128.8,128.9,129.2,133.8,137.1,137.2,{ }^{*} 138.6,138.6,{ }^{*} 140.9, * 141.0,141.5,141.6, *$ 158.1, 158.2,* 198.7 ppm; IR (KBr) 1685, 1447, 1307, 1148, $1084 \mathrm{~cm}^{-1}$; HRMS $\left(\mathrm{FAB}^{+}\right.$) calcd for $\mathrm{C}_{70} \mathrm{H}_{107} \mathrm{O}_{10} \mathrm{~S}_{3} \mathrm{Si}_{2}$ 1259.6565, found 1259.6553.

* peaks from the other diastereoisomer.

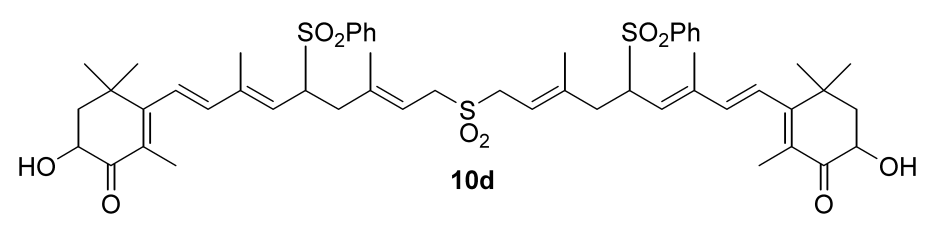

\section{Bis(5-benzenesulfonyl-3,7-dimethyl-9-(4-hydroxy-3-oxo-2,6,6-trimethyl-1-cyclohexenyl)-2,6,8-}

nonatrienyl) sulfone (10d). Following the general procedure for 10b, keto-trisulfone 7 (4.70 g, $5.13 \mathrm{mmol}$ ) reacted with trimethylsilyl trifluoromethanesulfonate $(2.04 \mathrm{~mL}, 11.29 \mathrm{mmol})$ in $\mathrm{CH}_{2} \mathrm{Cl}_{2}(80 \mathrm{~mL})$ under $\mathrm{Et}_{3} \mathrm{~N}$ $(3.58 \mathrm{~mL}, 25.65 \mathrm{mmol})$ at room temperature for $10 \mathrm{~min}$ to give $9 \mathrm{a}(5.91 \mathrm{~g})$ as a white solid.

The oxidation of $9 \mathbf{a}(5.91 \mathrm{~g})$ with perphthalic acid, which was prepared by the reaction of urea- $\mathrm{H}_{2} \mathrm{O}_{2}(\mathrm{UHP}$, $4.83 \mathrm{~g}, 51.30 \mathrm{mmol})$ and phthalic anhydride $(3.80 \mathrm{~g}, 25.65 \mathrm{mmol})$ in $\mathrm{CH}_{3} \mathrm{CN}(80 \mathrm{~mL})$, under $\mathrm{Na}_{2} \mathrm{CO}_{3}(5.44 \mathrm{~g}$, $51.30 \mathrm{mmol})$ in $\mathrm{CH}_{2} \mathrm{Cl}_{2}(50 \mathrm{~mL})$ at room temperature for $2.5 \mathrm{~h}$, followed by the deprotection of TMS group 
with a $50 \%$ HF solution $(4.4 \mathrm{~g}, 0.11 \mathrm{~mol})$ gave crude $10 \mathrm{~d}$ as a light-yellow solid. The crude product was purified by washing with $\mathrm{MeOH}$ to give pure $10 \mathrm{~d}(4.11 \mathrm{~g}, 4.33 \mathrm{mmol})$ in $85 \%$ yield, which was consisted of 1:1 diastereoisomers presumably at the carbons containing the hydroxy group.

Data for 10d: ${ }^{1}$ H NMR $\delta 1.10(\mathrm{~s}, 6 \mathrm{H}), 1.11$ (s, 6H), ${ }^{*} 1.24(\mathrm{~s}, 6 \mathrm{H}),{ }^{*} 1.28$ (s, 6H), 1.34 (s, 6H), 1.36 (s, 6H), 1.69 (s, 6H), 1.77 (dd, $J=14.1,2.9 \mathrm{~Hz}, 2 \mathrm{H}), 1.82(\mathrm{~s}, 6 \mathrm{H}),{ }^{*} 1.83(\mathrm{~s}, 6 \mathrm{H}), 2.15(\mathrm{ddd}, J=12.8,5.7,1.4 \mathrm{~Hz}, 2 \mathrm{H})$, $2.50(\mathrm{dd}, J=12.5,11.9 \mathrm{~Hz}, 2 \mathrm{H}), 3.04(\mathrm{~d}, J=13.9 \mathrm{~Hz}, 2 \mathrm{H}), 3.45-3.63(\mathrm{~m}, 4 \mathrm{H}), 3.66(\mathrm{br} \mathrm{s}, 2 \mathrm{H}), 4.09\left(\mathrm{dt}, J_{d}=\right.$ 3.7, $\left.J_{t}=10.5 \mathrm{~Hz}, 2 \mathrm{H}\right), 4.29(\mathrm{dd}, J=5.6,3.4 \mathrm{~Hz}, 2 \mathrm{H}), 4.34(\mathrm{dd}, J=5.6,3.4 \mathrm{~Hz}, 2 \mathrm{H}),{ }^{*} 5.25(\mathrm{~d}, J=10.5 \mathrm{~Hz}$, $2 \mathrm{H}), 5.28(\mathrm{br} \mathrm{s}, 2 \mathrm{H}), 6.04\left(\mathrm{~d}\right.$ of A of ABq, $\left.J_{A B}=16.3, J_{d}=2.8 \mathrm{~Hz}, 2 \mathrm{H}\right), 6.15\left(\mathrm{~d}\right.$ of B of ABq, $J_{A B}=16.3, J_{d}=$ $2.0 \mathrm{~Hz}, 2 \mathrm{H}), 7.47-7.56(\mathrm{~m}, 4 \mathrm{H}), 7.62-7.70(\mathrm{~m}, 2 \mathrm{H}), 7.76-7.85(\mathrm{~m}, 4 \mathrm{H}) \mathrm{ppm} ;{ }^{13} \mathbf{C}$ NMR $\delta 12.4,12.4,{ }^{*} 13.7$, $17.125 .9,25.9, * 30.4,30.5, * 36.7,37.7,37.7, * 45.2,51.5,63.4,69.2,113.9,125.3,125.3 * * 125.8,127.4$, $127.4,{ }^{*} 129.0,129.3,133.9,137.2,137.2, * 139.3,139.3, * 141.0,141.0, * 141.4,141.5,{ }^{*} 161.2,161.3, * 200.4$ ppm; IR (KBr) 3482, 1665, 1447, 1305, 1146, 913, $743 \mathrm{~cm}^{-1}$; Anal. Calcd for $\mathrm{C}_{52} \mathrm{H}_{66} \mathrm{O}_{10} \mathrm{~S}_{3}$ : C, 65.93; H, 7.02; S, 10.16. Found: C, 65.79; H, 7.01; S, 10.35.

* peaks from the other diastereoisomer.

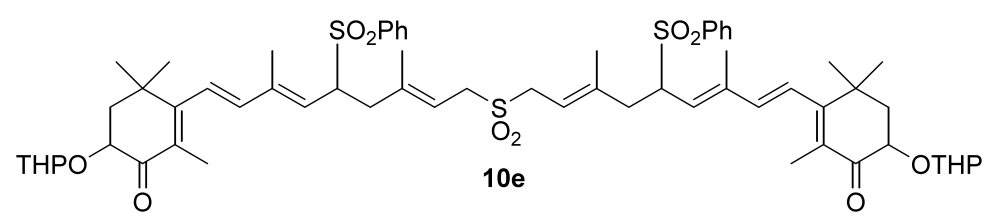

Bis(5-benzenesulfonyl-3,7-dimethyl-9-(3-oxo-4-tetrahydropyranyloxy-2,6,6-trimethyl-1-

cyclohexenyl)-2,6,8-nonatrienyl) sulfone (10e). To a stirred solution of hydroxy-keto-trisulfone 10d (1.93 g, $2.04 \mathrm{mmol})$ in $\mathrm{CH}_{2} \mathrm{Cl}_{2}(50 \mathrm{~mL})$ were added 3,4-dihydro- $2 \mathrm{H}$-pyrane (DHP, $0.69 \mathrm{~mL}, 8.15 \mathrm{mmol}$ ) and 10- 
camphorsulfonic acid (CSA, $0.19 \mathrm{~g}, 0.82 \mathrm{mmol}$ ). The mixture was stirred at room temperature for $30 \mathrm{~min}$, diluted with $\mathrm{CH}_{2} \mathrm{Cl}_{2}$, washed with a saturated aqueous $\mathrm{NaHCO}_{3}$ solution, dried over anhydrous $\mathrm{K}_{2} \mathrm{CO}_{3}$, filtered, and concentrated under reduced pressure. The crude product was purified by flash column chromatography on silica gel which was deactivated by $\mathrm{Et}_{3} \mathrm{~N}$ to give $\mathbf{1 0 e}(1.98 \mathrm{~g}, 1.78 \mathrm{mmol})$ in $87 \%$ yield as a white crystalline solid, which was presumably consisted of diastereoisomers with $\sim 1: 1$ and 3:1 ratios at the carbons containing the etheral group and at the acetal carbons, respectively.

Data for the major stereoisomers of 10e: ${ }^{1}$ H NMR $\delta 1.10(\mathrm{~s}, 6 \mathrm{H}),{ }^{*} 1.11(\mathrm{~s}, 6 \mathrm{H}), 1.23(\mathrm{~s}, 6 \mathrm{H}), 1.27(\mathrm{~s}, 6 \mathrm{H}){ }^{*}$ $1.32(\mathrm{~s}, 6 \mathrm{H}),{ }^{*} 1.35(\mathrm{~s}, 6 \mathrm{H}), 1.50-1.68(\mathrm{~m}, 6 \mathrm{H}), 1.69(\mathrm{~s}, 6 \mathrm{H}), 1.76(\mathrm{~s}, 6 \mathrm{H}), 1.77(\mathrm{~s}, 6 \mathrm{H}),{ }^{*} 1.80-2.09(\mathrm{~m}, 10 \mathrm{H})$, $2.49(\mathrm{dd}, J=11.0,10.1 \mathrm{~Hz}, 2 \mathrm{H}), 3.04(\mathrm{~d}, J=14.0 \mathrm{~Hz}, 2 \mathrm{H}), 3.42-3.62(\mathrm{~m}, 6 \mathrm{H}), 3.90(\mathrm{dd}, J=9.7,8.4 \mathrm{~Hz}, 2 \mathrm{H})$, $4.09(\mathrm{dd}, J=11.4,9.7 \mathrm{~Hz}, 2 \mathrm{H}), 4.18(\mathrm{~m}, 2 \mathrm{H}){ }^{*} 4.44(\mathrm{dd}, J=6.2,2.6 \mathrm{~Hz}, 2 \mathrm{H}), 4.48(\mathrm{dd}, J=6.0,2.9 \mathrm{~Hz}, 2 \mathrm{H}){ }^{*}$ $4.83($ br s, $2 \mathrm{H}){ }^{*} 5.03($ br s, $2 \mathrm{H}), 5.21(\mathrm{~d}, J=10.7 \mathrm{~Hz}, 2 \mathrm{H}), 5.28(\mathrm{t}, J=6.8 \mathrm{~Hz}, 2 \mathrm{H}), 6.03\left(\mathrm{~d}\right.$ of $\mathrm{A}$ of $\mathrm{ABq}, J_{A B}$ $\left.=16.4, J_{d}=3.4 \mathrm{~Hz}, 2 \mathrm{H}\right), 6.11\left(\mathrm{~d}\right.$ of B of ABq, $\left.J_{A B}=16.4, J_{d}=2.7 \mathrm{~Hz}, 2 \mathrm{H}\right), 7.47-7.56(\mathrm{~m}, 4 \mathrm{H}), 7.61-7.69(\mathrm{~m}$, 2H), 7.77-7.84 (m, 4H) ppm; ${ }^{13}$ C NMR $\delta 12.3, * 12.4,13.7,17.1,19.5,25.5,26.3, * 26.4,30.2,30.3, * 30.5$, $37.0,37.6, * 37.7,44.5, * 44.5,51.4,61.6,62.7, * 63.4,72.2,72.2 *{ }^{*} 99.2,99.2, * 113.9,124.8,124.9$, ${ }^{*} 126.2$, $128.9,129.2,129.3,129.4, * 133.9,137.2,138.8,138.8 *{ }^{*} 141.0,141.0, * 141.5, * 141.6,159.3,159.4, * 199.0$ ppm; IR (KBr) 1677, 1447, 1306, 1147, 1084, 996, $734 \mathrm{~cm}^{-1} ; \mathbf{H R M S}\left(\mathrm{FAB}^{+}\right)$calcd for $\mathrm{C}_{46} \mathrm{H}_{62} \mathrm{O}_{8} \mathrm{~S}_{2}$ $\left(\mathrm{C}_{62} \mathrm{H}_{83} \mathrm{O}_{12} \mathrm{~S}_{3}-\left(2 \mathrm{C}_{5} \mathrm{H}_{8} \mathrm{O}+\mathrm{C}_{6} \mathrm{H}_{5} \mathrm{O}_{2} \mathrm{~S}\right)\right)$ 806.3886, found 806.3878.

*: peaks from the $2^{\text {nd }}$ major diastereoisomer 


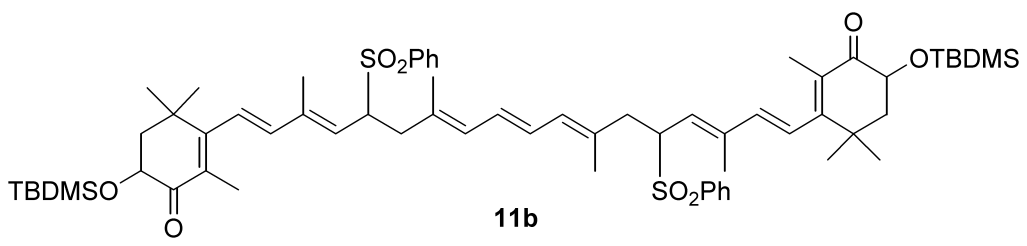

\section{1,18-Bis(3-oxo-4-(t-butyldimethylsiloxy)-2,6,6-trimethyl-1-cyclohexenyl)-5,14-dibenzenesulfonyl-}

3,7,12,16-tetramethyl-1,3,7,9, 11, 15, 17-eicosaheptaene (11b). Following the general procedure for 8 , the reaction of $\mathrm{C}_{40}$ keto-trisulfone compound $\mathbf{1 0 b}(663 \mathrm{mg}, 0.56 \mathrm{mmol})$ with $\mathrm{NaOCH}_{3}(305 \mathrm{mg}, 5.65 \mathrm{mmol})$ and $\mathrm{CCl}_{4}(6 \mathrm{~mL})$ in $\mathrm{CH}_{2} \mathrm{Cl}_{2}(6 \mathrm{~mL})$ at room temperature for $3.5 \mathrm{~h}$ gave $\mathbf{1 1 b}(173 \mathrm{mg}, 0.18 \mathrm{mmol})$ in $32 \%$ yield as a mixture of diastereoisomers after purification by column chromatography on silica gel which was deactivated by $\mathrm{Et}_{3} \mathrm{~N}$.

Data for the major stereoisomer of 11b: ${ }^{1} \mathbf{H}$ NMR $\delta 0.09(\mathrm{~s}, 6 \mathrm{H}), 0.18(\mathrm{~s}, 6 \mathrm{H}), 0.93(\mathrm{~s}, 18 \mathrm{H}), 1.09(\mathrm{~s}, 6 \mathrm{H})$, $1.20(\mathrm{~s}, 6 \mathrm{H}), 1.26(\mathrm{~s}, 6 \mathrm{H}), 1.68(\mathrm{~s}, 6 \mathrm{H}), 1.78(\mathrm{~s}, 6 \mathrm{H}), 1.90-2.00(\mathrm{~m}, 4 \mathrm{H}), 2.44(\mathrm{dd}, J=13.2,9.2 \mathrm{~Hz}, 2 \mathrm{H}), 3.03$ $(\mathrm{dd}, J=13.2,8.8 \mathrm{~Hz}, 2 \mathrm{H}), 4.05(\mathrm{dd}, J=9.2,8.8 \mathrm{~Hz}, 2 \mathrm{H}), 4.25-4.37(\mathrm{~m}, 2 \mathrm{H}), 5.24(\mathrm{~d}, J=10.3 \mathrm{~Hz}, 2 \mathrm{H}), 5.88$ (br s, 2H), 5.93-6.16 (m, 4H), 6.20 (br s, 2H), 7.46-7.55 (m, 4H), 7.60-7.67 (m, 2H), 7.77-7.86 (m, 4H) ppm; ${ }^{13} \mathbf{C}$ NMR $\delta$-5.4, -4.5, 12.3, 13.8, 16.5, 18.5, 25.8, 26.2, 30.3, 36.8, 38.0, 46.8, 63.9, 71.0, 125.3, 125.6, 127.8, 128.7, 128.8, 129.2, 132.8, 132.9, 133.7, 137.5, 139.1, 141.1, 159.0, 198.8 ppm; IR (KBr) 1683, 1447, 1306, 1148, $1085 \mathrm{~cm}^{-1}$; HRMS $\left(\mathrm{FAB}^{+}\right)$calcd for $\mathrm{C}_{64} \mathrm{H}_{93} \mathrm{O}_{8} \mathrm{~S}_{2} \mathrm{Si}_{2} 1109.5850$, found 1109.5829 .

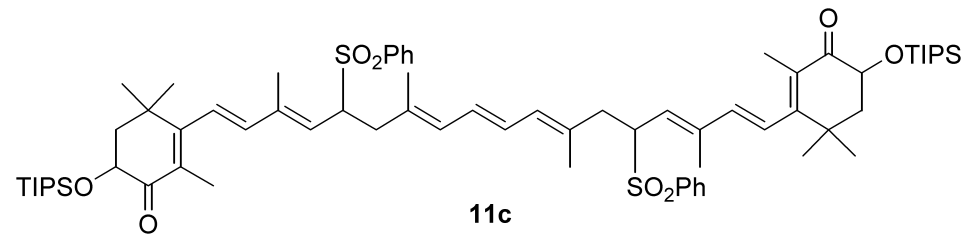

1,18-bis(3-oxo-4-triisopropylsiloxy-2,6,6-trimethyl-1-cyclohexenyl)-5,14-dibenzenesulfonyl-3,7,12,16-

tetramethyl-1,3,7,9,11,15,17-eicosahepataene (11c). Following the general procedure for 8 , the reaction of 
$\mathrm{C}_{40}$ keto-trisulfone compound $\mathbf{1 0 c}(3.09 \mathrm{~g}, 2.45 \mathrm{mmol})$ with $\mathrm{NaOCH}_{3}(1.32 \mathrm{~g}, 24.5 \mathrm{mmol})$ and $\mathrm{CCl}_{4}(25 \mathrm{~mL})$ in $\mathrm{CH}_{2} \mathrm{Cl}_{2}(25 \mathrm{~mL})$ at room temperature for $4 \mathrm{~h}$ gave $11 \mathrm{c}(2.25 \mathrm{~g}, 1.88 \mathrm{mmol})$ in $77 \%$ yield, that was consisted of $\sim 1: 1$ diastereoisomers presumably at the carbons containing the etheral group, after purification by column chromatography on silica gel which was deactivated by $\mathrm{Et}_{3} \mathrm{~N}$.

Data for 11c: ${ }^{1}$ H NMR $\delta 1.11(\mathrm{~s}, 42 \mathrm{H}), 1.19(\mathrm{~s}, 6 \mathrm{H}), 1.20(\mathrm{~s}, 6 \mathrm{H}),{ }^{*} 1.22(\mathrm{~s}, 6 \mathrm{H}),{ }^{*} 1.24(\mathrm{~s}, 6 \mathrm{H}), 1.30(\mathrm{~s}, 6 \mathrm{H})$, $1.32(\mathrm{~s}, 6 \mathrm{H}),{ }^{*} 1.68(\mathrm{~s}, 6 \mathrm{H}), 1.78(\mathrm{~s}, 6 \mathrm{H}), 1.79(\mathrm{~s}, 6 \mathrm{H}),{ }^{*} 1.99(\mathrm{~d}, J=7.8 \mathrm{~Hz}, 4 \mathrm{H}), 2.32-2.55(\mathrm{~m}, 2 \mathrm{H}), 2.88-3.15$ $(\mathrm{m}, 2 \mathrm{H}), 4.05$ (br t, $J=10.6 \mathrm{~Hz}, 2 \mathrm{H}), 4.35-4.50(\mathrm{~m}, 2 \mathrm{H}), 5.24(\mathrm{~d}, J=10.1 \mathrm{~Hz}, 2 \mathrm{H}), 5.87$ (br s, 2H), $6.00(\mathrm{~d}$ of A of $\left.\mathrm{ABq}, J_{A B}=16.2, J_{d}=6.5 \mathrm{~Hz}, 2 \mathrm{H}\right), 6.12\left(\mathrm{br} \mathrm{B}\right.$ of ABq, $\left.J_{A B}=16.2 \mathrm{~Hz}, 2 \mathrm{H}\right), 6.20(\mathrm{br} \mathrm{s}, 2 \mathrm{H}), 7.43-7.68(\mathrm{~m}$, 6H), 7.76-8.07 (m, 4H) ppm; ${ }^{13} \mathbf{C}$ NMR (major stereoisomer) $\delta$ 12.4, 12.4, 14.0, 16.6, 18.0, 18.0, 26.3, 30.3, 36.9, 38.1, 47.1, 64.2, 70.9, 125.3, 125.7, 127.9, 128.9, 128.9, 129.3, 132.8, 133.0, 133.7, 137.5, 139.1, 141.1, 158.4, 198.8 ppm; IR (KBr) 1686, 1447, 1306, 1147, $1084 \mathrm{~cm}^{-1}$; HRMS (FAB ${ }^{+}$) calcd for $\mathrm{C}_{70} \mathrm{H}_{105} \mathrm{O}_{8} \mathrm{~S}_{2} \mathrm{Si}_{2}$ 1193.6789, found 1193.6804 .

* peaks from the other diastereoisomer.

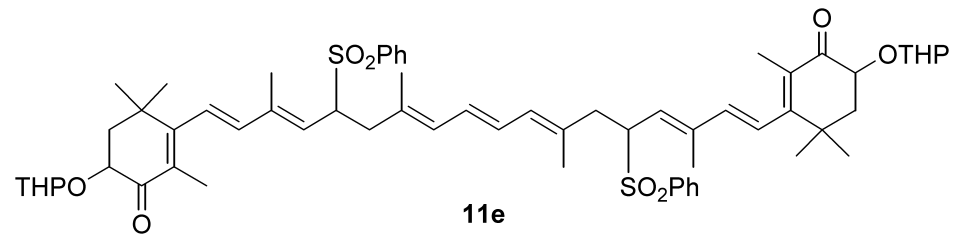

1,18-Bis(3-oxo-4-tetrahydropyranyloxy-2,6,6-trimethyl-1-cyclohexenyl)-5,14-dibenzenesulfonyl-

3,7,12,16-tetramethyl-1,3,7,9,11,15,17-eicosahepataene (11e). Following the general procedure for 8 , the reaction of $\mathrm{C}_{40}$ keto-trisulfone compound $10 \mathrm{e}(0.96 \mathrm{~g}, 0.86 \mathrm{mmol})$ with $\mathrm{NaOCH}_{3}(0.56 \mathrm{~g}, 10.38 \mathrm{mmol})$ and $\mathrm{CCl}_{4}(20 \mathrm{~mL})$ in $\mathrm{CH}_{2} \mathrm{Cl}_{2}(30 \mathrm{~mL})$ at room temperature for $3 \mathrm{~h}$ gave 11e $(0.84 \mathrm{~g}, 0.80 \mathrm{mmol})$ in $93 \%$ crude 
yield as a yellow crystalline solid. The crude product, which contained diastereoisomers with $\sim 1: 1$ and 3:1 ratios at the carbons containing the etheral group and at the acetal carbons, respectively, was not stable under silica gel chromatographic condition, but was pure enough to be used in the next dehydrosulfonation step without further purification.

Data for the major stereoisomer of 11e: ${ }^{1} \mathbf{H}$ NMR $\delta 1.12(\mathrm{~s}, 6 \mathrm{H}), 1.23(\mathrm{~s}, 6 \mathrm{H}), 1.30(\mathrm{~s}, 6 \mathrm{H}), 1.48-1.65(\mathrm{~m}$, 6H), $1.70(\mathrm{~s}, 6 \mathrm{H}), 1.78(\mathrm{~s}, 6 \mathrm{H}), 1.80-2.10(\mathrm{~s}, 10 \mathrm{H}), 2.44(\mathrm{dd}, J=13.4,10.8 \mathrm{~Hz}, 2 \mathrm{H}), 3.02(\mathrm{~d}, J=13.7 \mathrm{~Hz}$, 2H), 3.52 (br s, 2H), 3.89 (br s, 2H), 4.05 (br t, $J=9.5 \mathrm{~Hz}, 2 \mathrm{H}), 4.43(\mathrm{dd}, J=6.4,2.9 \mathrm{~Hz}, 2 \mathrm{H}), 5.02(\mathrm{br} \mathrm{s}, 2 \mathrm{H})$, $5.24(\mathrm{~d}, J=11.8 \mathrm{~Hz}, 2 \mathrm{H}), 5.87(\mathrm{br} \mathrm{s}, 2 \mathrm{H}), 5.93-6.15(\mathrm{~m}, 4 \mathrm{H}), 6.21(\mathrm{br} \mathrm{s}, 2 \mathrm{H}), 7.40-8.00(\mathrm{~m}, 10 \mathrm{H}) \mathrm{ppm} ;{ }^{13} \mathbf{C}$ NMR $\delta 12.3,13.6,16.5,19.5,25.5,26.4,30.2,30.4,36.9,38.1,44.5,62.7,64.0,72.2,99.2,123.9,125.5$, $127.8,128.8,128.8,129.2,132.8,133.7,137.5,139.2,141.0,144.5,159.6,199.0$ ppm; IR (KBr) 1677, 1447 , 1306, 1146, 1083, 1032, $732 \mathrm{~cm}^{-1}$; HRMS $\left(\mathrm{FAB}^{+}\right)$calcd for $\mathrm{C}_{52} \mathrm{H}_{65} \mathrm{O}_{8} \mathrm{~S}_{2}\left(\mathrm{C}_{62} \mathrm{H}_{81} \mathrm{O}_{10} \mathrm{~S}_{2}-2 \mathrm{C}_{5} \mathrm{H}_{8} \mathrm{O}\right) 881.4121$, found 881.4106 .

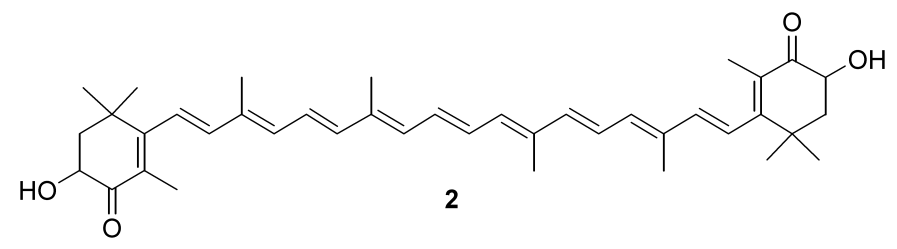

Astaxanthin (2). Following the general procedure for 1, $\mathrm{C}_{40}$ keto-disulfone 11e $(0.23 \mathrm{~g}, 0.22 \mathrm{mmol})$ in benzene $(10 \mathrm{~mL})$ reacted with $\mathrm{NaOEt}$, that was prepared by the reaction of $\mathrm{Na}(98 \mathrm{mg}, 4.26 \mathrm{mmol})$ and 99.9\% EtOH (20 mL), at reflux for $16 \mathrm{~h}$ to give THP-protected astaxanthin $(0.14 \mathrm{~g}, 0.18 \mathrm{mmol})$ in $83 \%$ crude yield.

THP-protected astaxanthin $(0.14 \mathrm{~g}, 0.18 \mathrm{mmol})$ then reacted with $\mathrm{MeOH}(10 \mathrm{~mL})$ and $p$-toluenesulfonic 
acid ( $p$ - $\mathrm{TsOH}, 34 \mathrm{mg}, 0.18 \mathrm{mmol})$ in $\mathrm{CH}_{2} \mathrm{Cl}_{2}(30 \mathrm{~mL})$ at room temperature for $4 \mathrm{~h}$ to give crude astaxanthin.

The crude product was recrystallized from acetone/MeOH to give pure $2(0.048 \mathrm{~g}, 0.08 \mathrm{mmol})$, and the mother liquor was purified again by silica gel column chromatography to give $2(0.030 \mathrm{~g}, 0.05 \mathrm{mmol})$. The combined yield of $2(0.078 \mathrm{~g}, 0.13 \mathrm{mmol})$ in the deprotection step was $72 \%$, and the overall yield of astaxanthin (2) was 60\% from $11 \mathrm{e}$.

Data for 2: ${ }^{1} \mathbf{H}$ NMR $\delta 1.21(6 \mathrm{H}, \mathrm{s}), 1.32(6 \mathrm{H}, \mathrm{s}), 1.81(2 \mathrm{H}, \mathrm{dd}, J=13.4,13.2 \mathrm{~Hz}), 1.94(6 \mathrm{H}, \mathrm{s}), 1.99(6 \mathrm{H}$, s), $2.00(6 \mathrm{H}, \mathrm{s}), 2.16(2 \mathrm{H}, \mathrm{dd}, J=13.2,5.5 \mathrm{~Hz}), 3.68(2 \mathrm{H}, \mathrm{d}, J=1.8 \mathrm{~Hz}), 4.32(2 \mathrm{H}, \mathrm{ddd}, J=13.4,5.5,1.8$ $\mathrm{Hz}), 6.22\left(2 \mathrm{H}, \mathrm{A}\right.$ of ABq, $\left.J_{A B}=16.0 \mathrm{~Hz}\right), 6.26-6.34(2 \mathrm{H}, \mathrm{m}), 6.30(2 \mathrm{H}, \mathrm{d}, J=11.5 \mathrm{~Hz}), 6.43(2 \mathrm{H}, \mathrm{B}$ of ABq, $\left.J_{A B}=16.0 \mathrm{~Hz}\right), 6.45(2 \mathrm{H}, \mathrm{d}, J=15.0 \mathrm{~Hz}), 6.65(2 \mathrm{H}, \mathrm{dd}, J=15.0,11.5 \mathrm{~Hz}), 6.63-6.70(2 \mathrm{H}, \mathrm{m}) \mathrm{ppm} ;{ }^{13} \mathbf{C}$ NMR $\delta 12.6,12.8,14.0,26.1,30.7,36.8,45.4,69.2,123.3,124.6,126.8,130.7,133.8,134.6,135.2,136.7$, 139.7, 142.3, 162.2, $200.4 \mathrm{ppm}$.

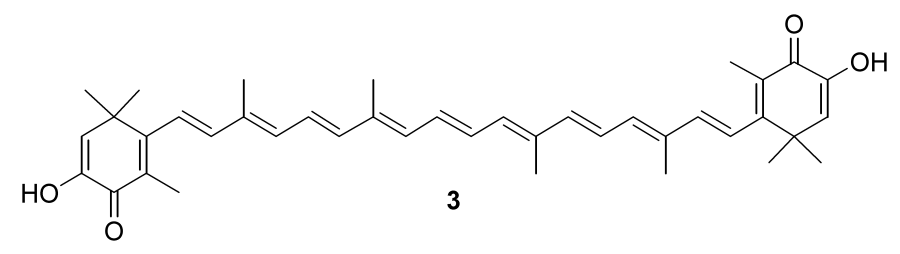

Astacene (3). Following the general procedure for 1, $\mathrm{C}_{40}$ keto-disulfone $11 \mathrm{c}(0.70 \mathrm{~g}, 0.59 \mathrm{mmol})$ in benzene $(10 \mathrm{~mL})$ reacted with $\mathrm{NaOEt}$, that was prepared by the reaction of $\mathrm{Na}(270 \mathrm{mg}, 11.73 \mathrm{mmol})$ and $99.9 \%$ EtOH $(20 \mathrm{~mL})$, at reflux for $19 \mathrm{~h}$ to give crude astacene. The crude product was purified by silica gel flash column chromatography to give $\mathbf{3}$ (250 $\mathrm{mg}, 0.42 \mathrm{mmol}$ ) in $71 \%$ yield as a dark-red solid.

Data for 3: ${ }^{1} \mathrm{H}$ NMR $\delta 1.30(12 \mathrm{H}, \mathrm{s}), 1.59(2 \mathrm{H}, \mathrm{s}), 2.00(6 \mathrm{H}, \mathrm{s}), 2.03(6 \mathrm{H}, \mathrm{s}), 2.10(6 \mathrm{H}, \mathrm{s}), 6.06(2 \mathrm{H}, \mathrm{s})$, $6.31(2 \mathrm{H}, \mathrm{d}, J=15.9 \mathrm{~Hz}), 6.34(2 \mathrm{H}, \mathrm{d}, J=10.7 \mathrm{~Hz}), 6.43(2 \mathrm{H}, \mathrm{s}), 6.48\left(2 \mathrm{H}, \mathrm{A}\right.$ of ABq, $\left.J_{A B}=15.6 \mathrm{~Hz}\right), 6.52$ 
$\left(2 \mathrm{H}, \mathrm{B}\right.$ of $\left.\mathrm{ABq}, J_{A B}=15.6 \mathrm{~Hz}\right), 6.68(2 \mathrm{H}, \mathrm{dd}, J=15.9,10.7 \mathrm{~Hz}) \mathrm{ppm} ;{ }^{13} \mathbf{C} \mathbf{N M R} \delta 12.6,12.8,13.7,28.2,28.2$, $39.3,123.1,124.6,125.5,128.3,130.7,133.9,134.7,135.4,136.8,139.8,142.5,144.5,161.4,182.5 \mathrm{ppm}$; IR (KBr) $3398,1624,1247,1064,968 \mathrm{~cm}^{-1}$. 


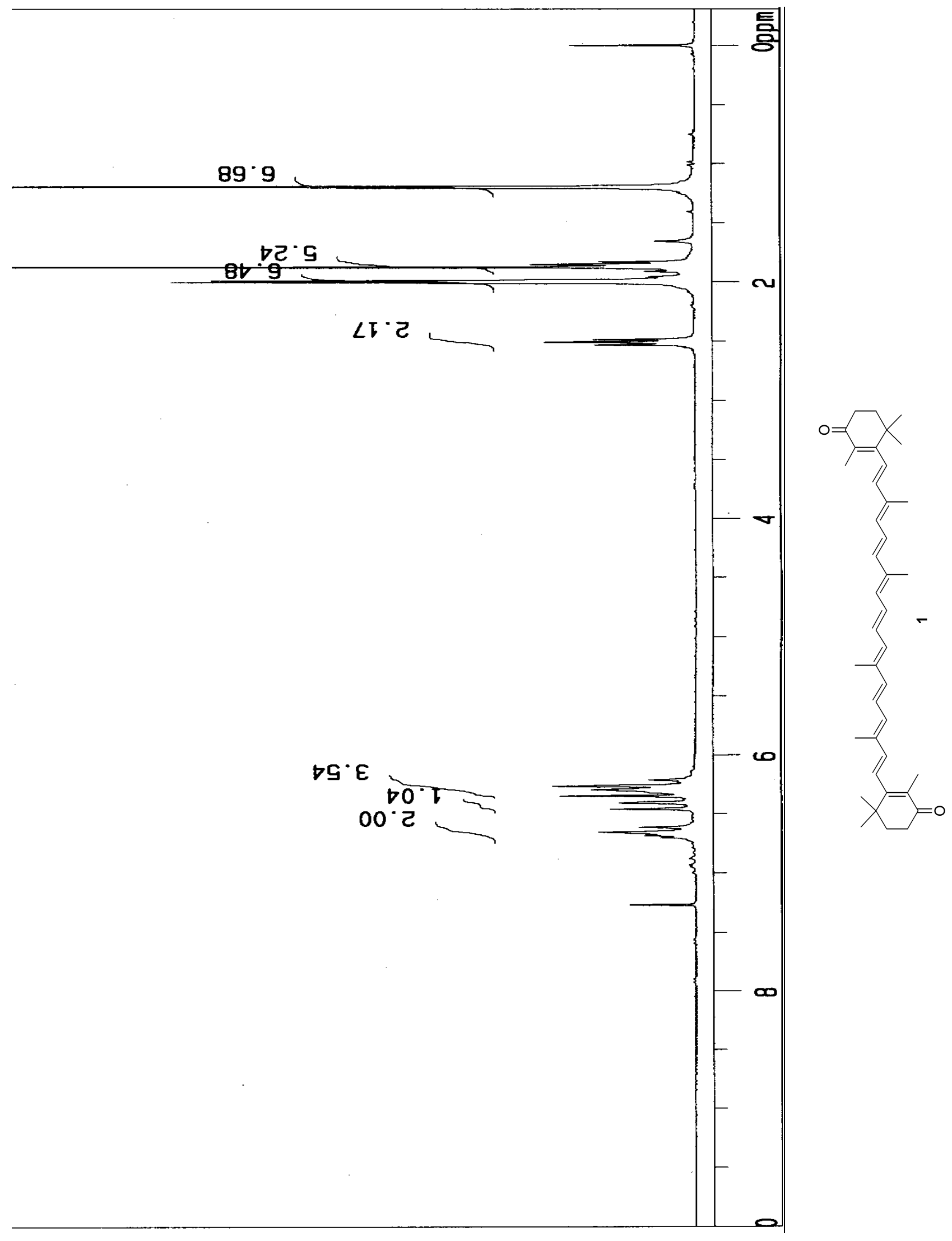



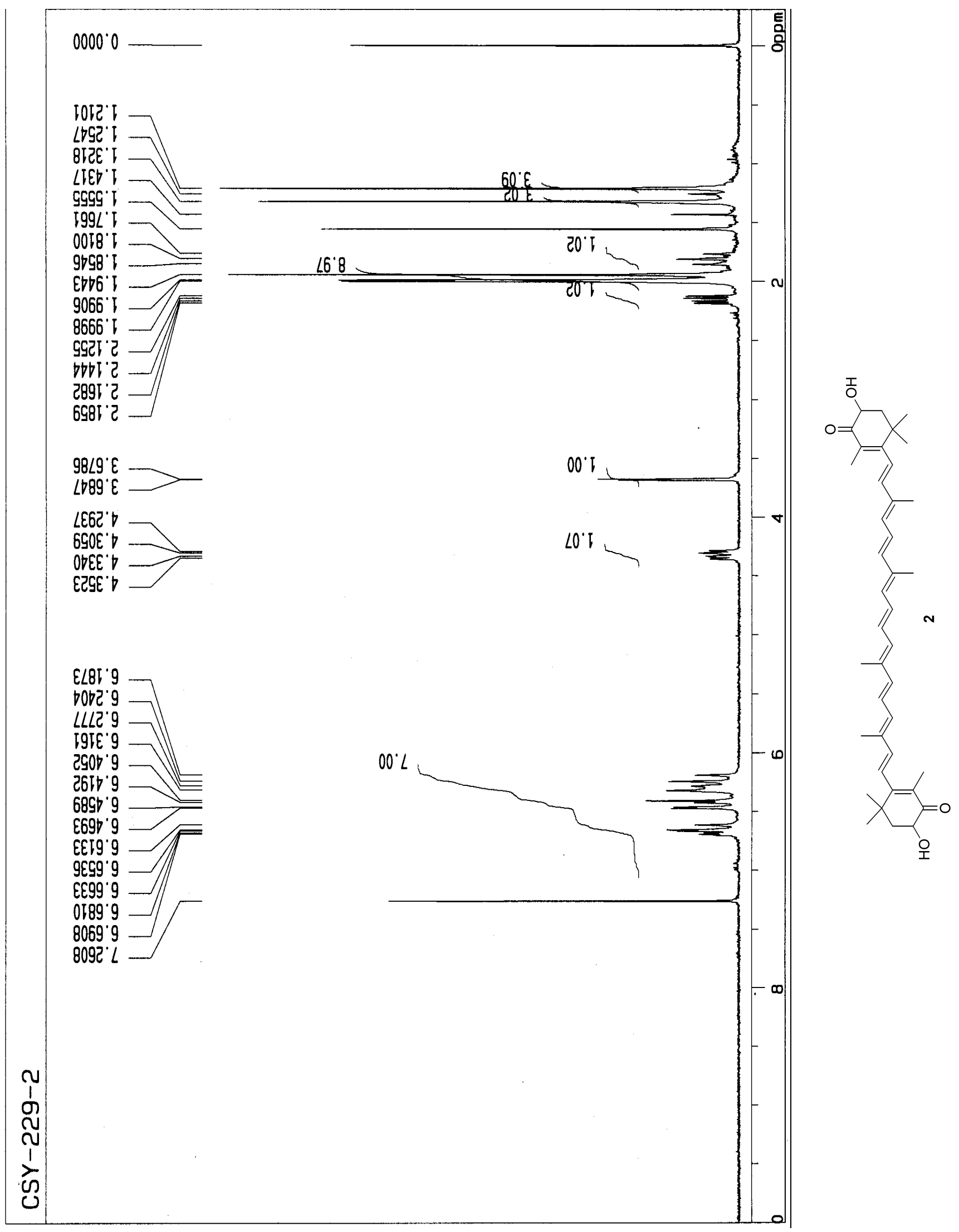


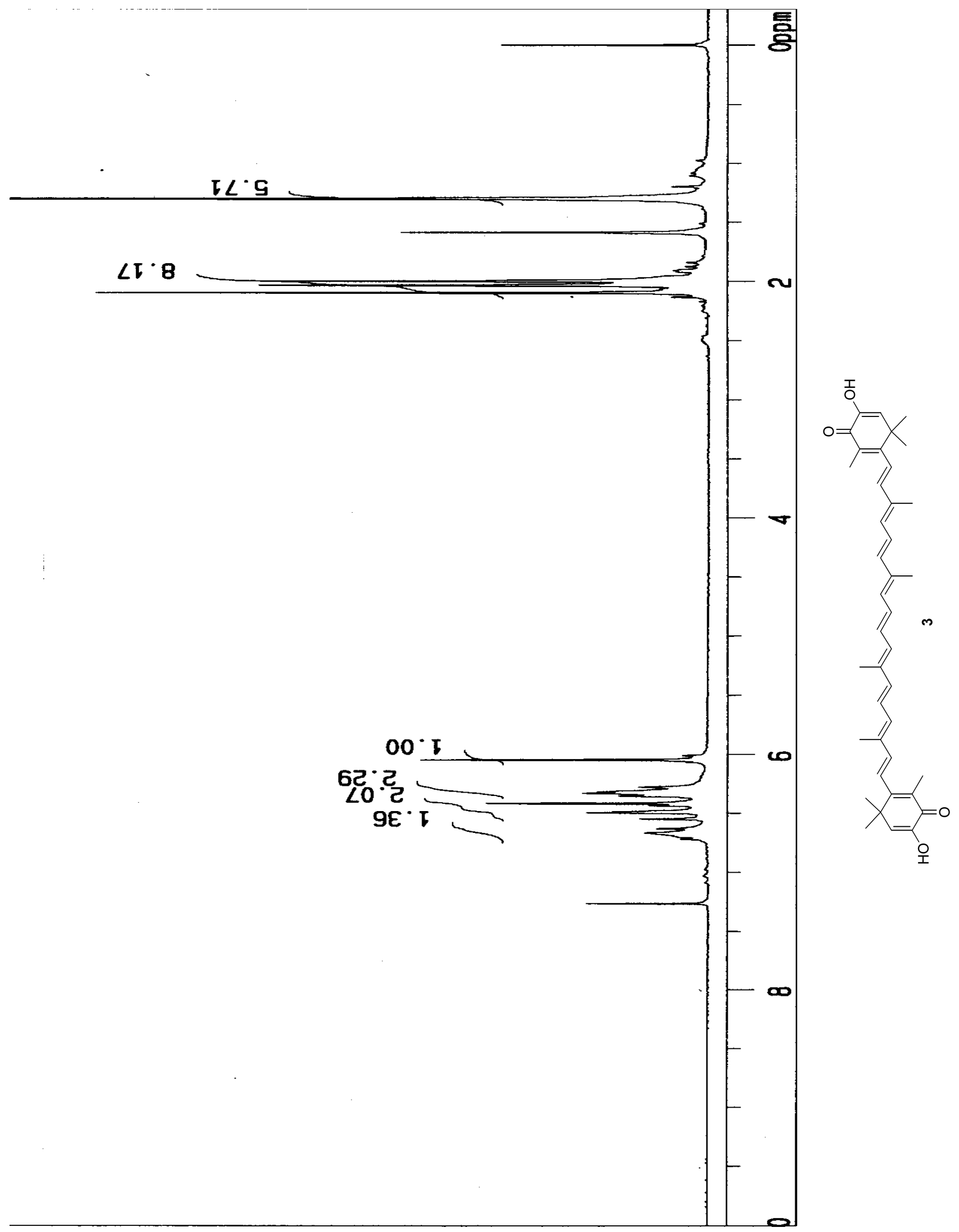




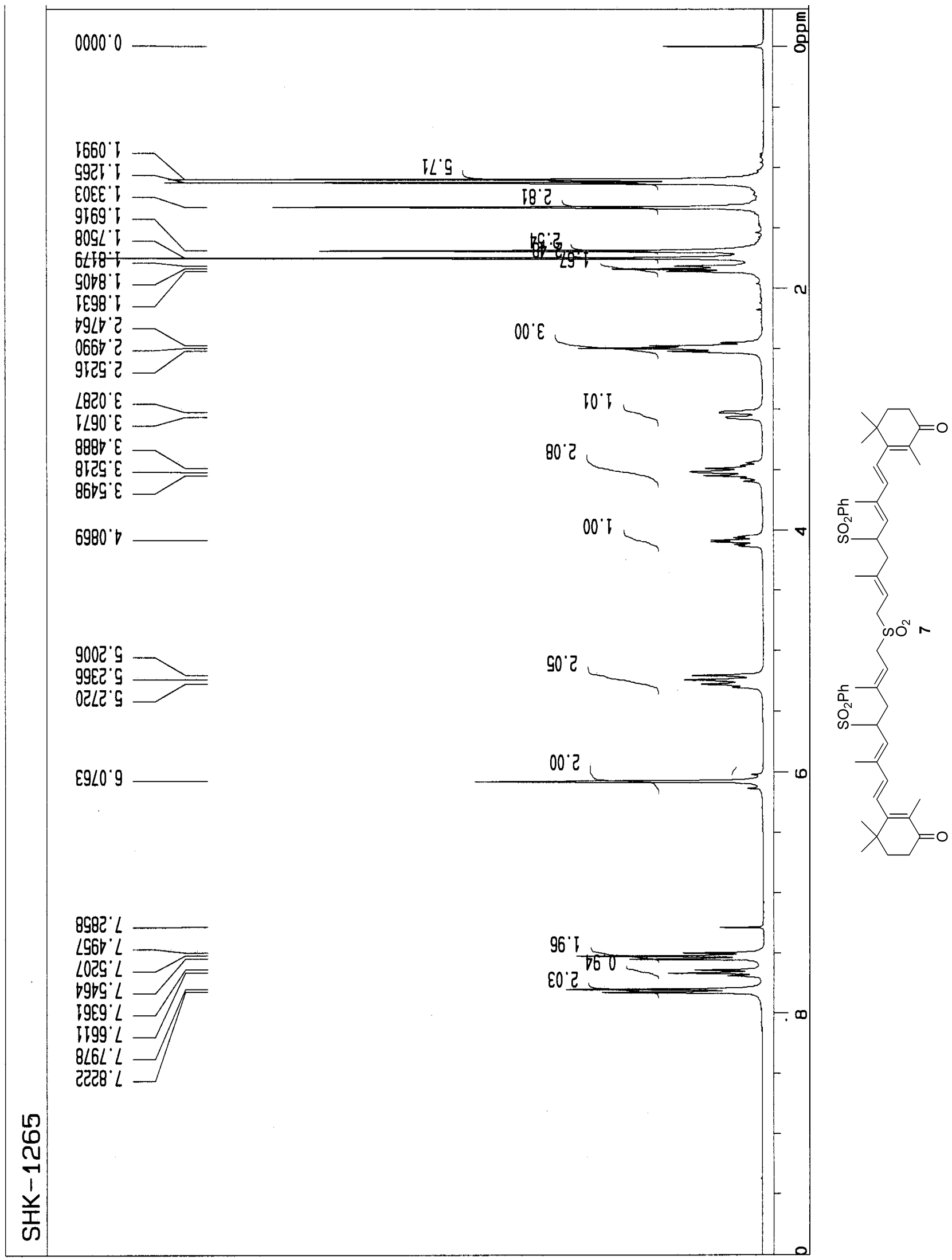




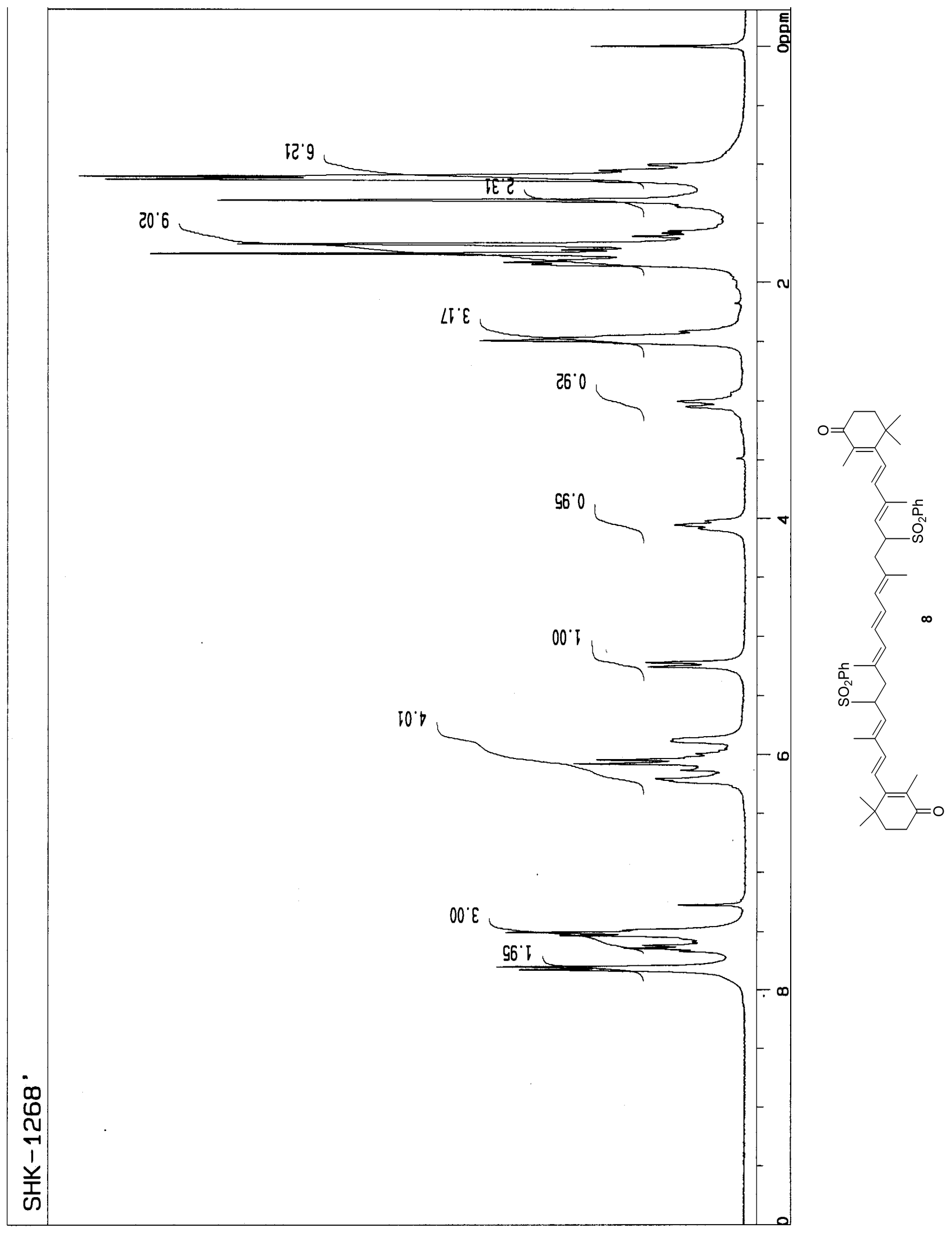




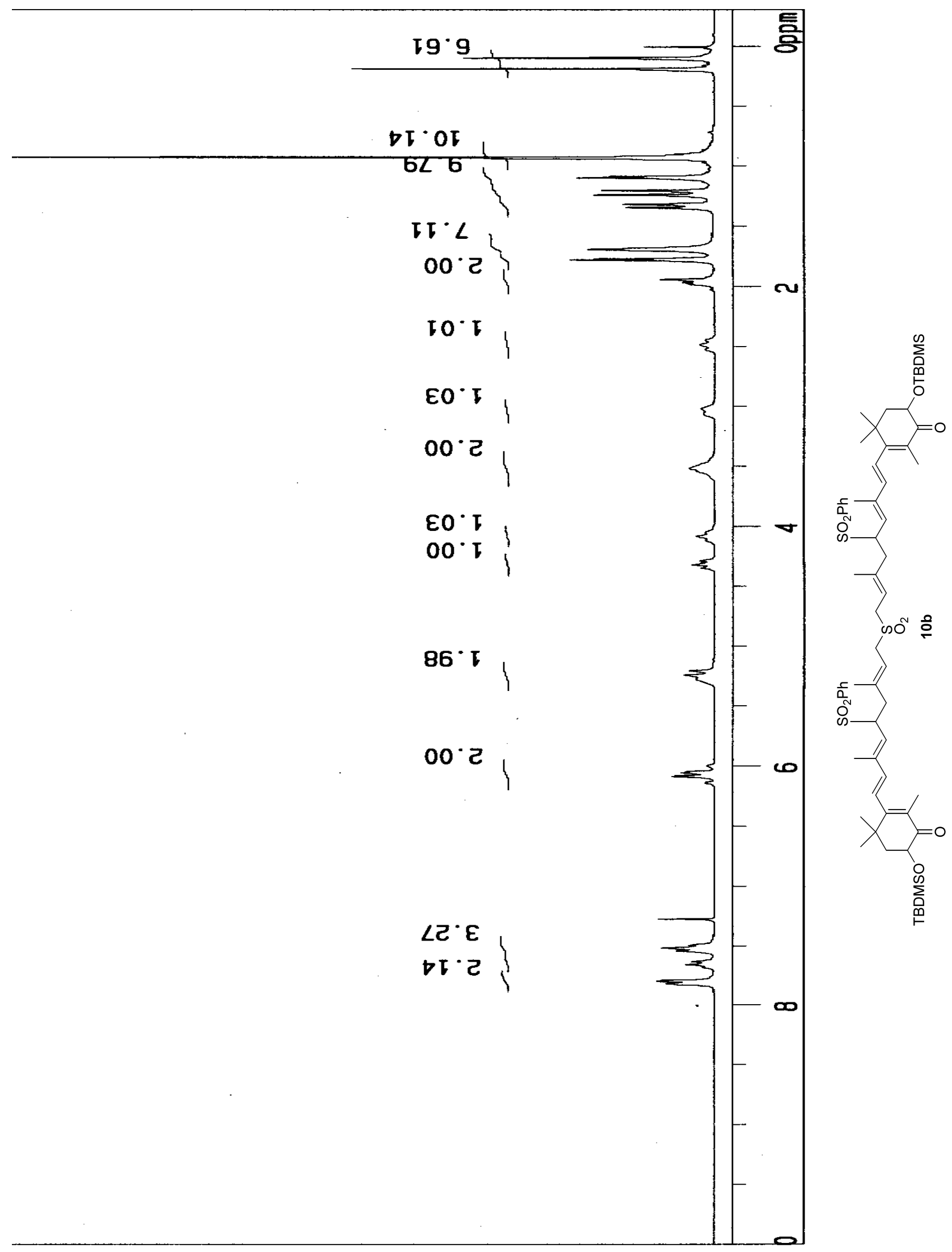




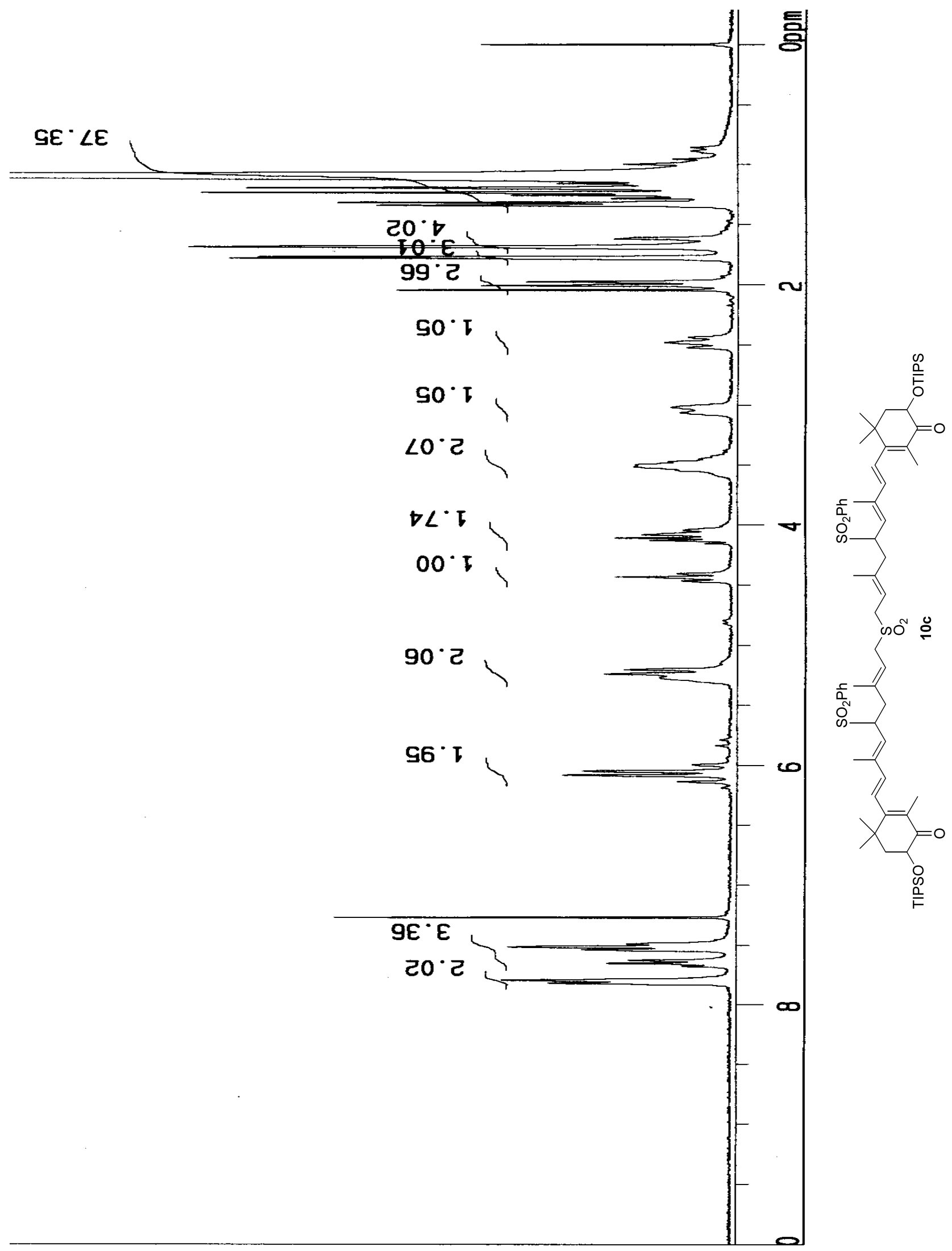




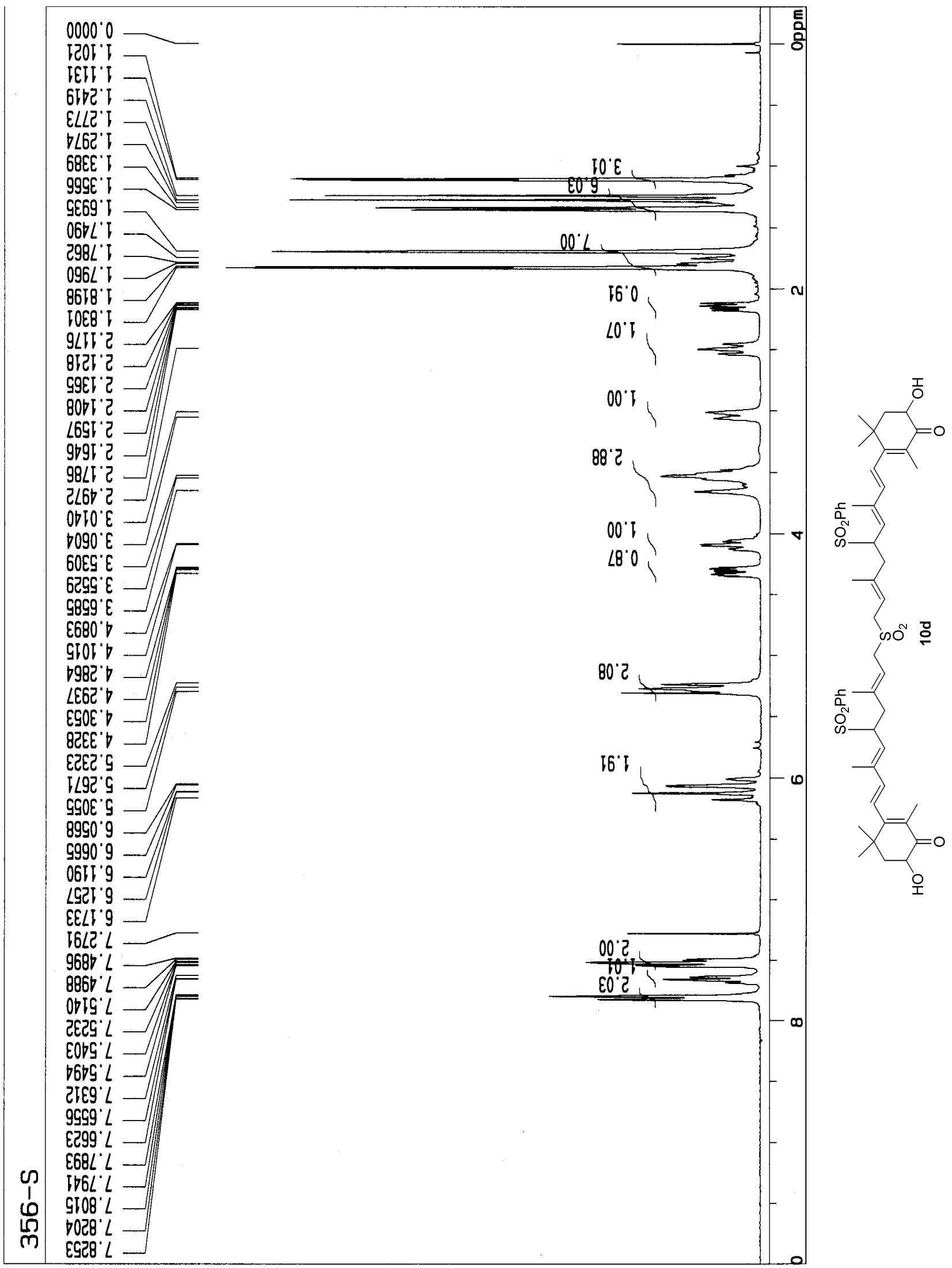



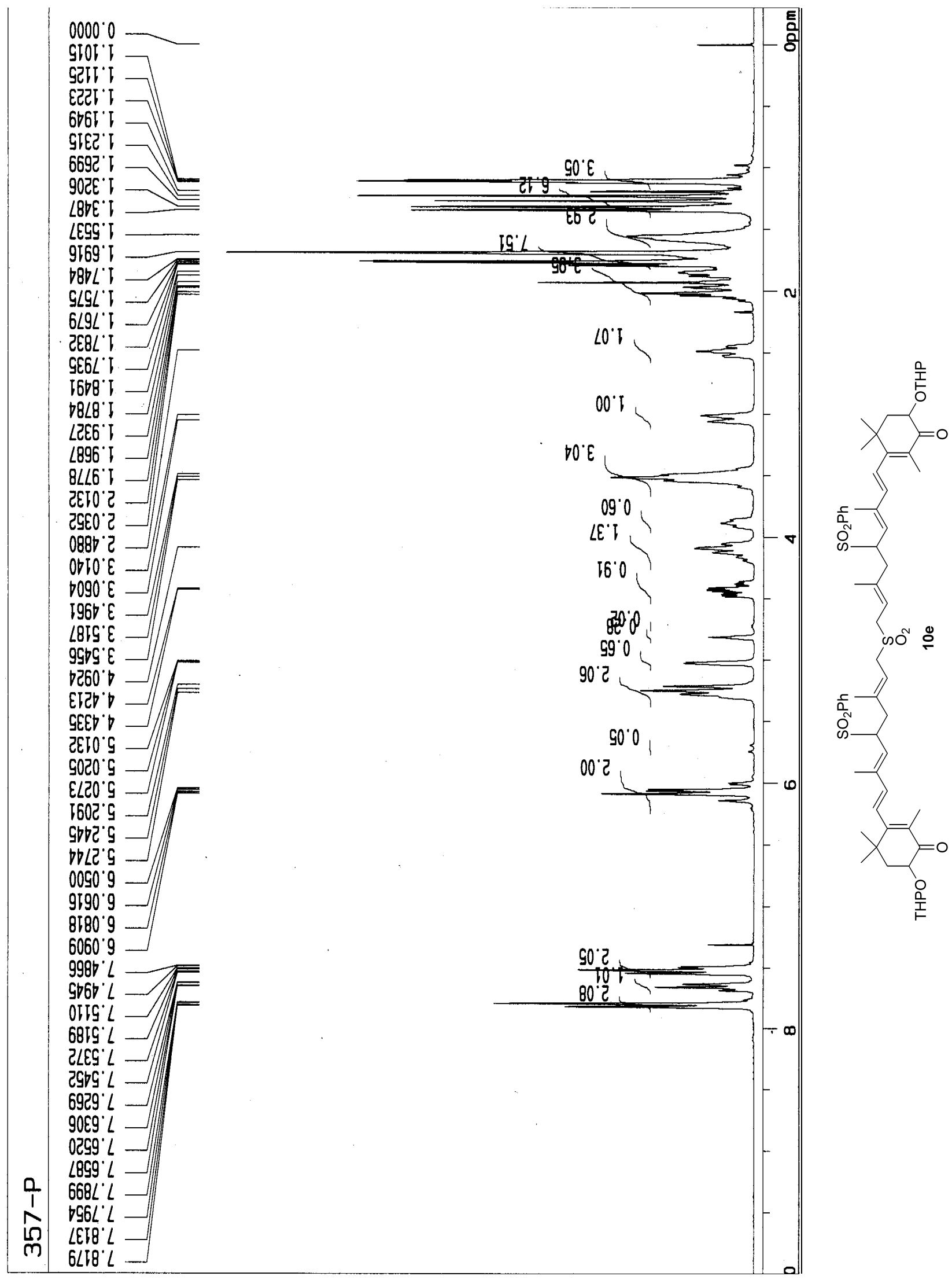

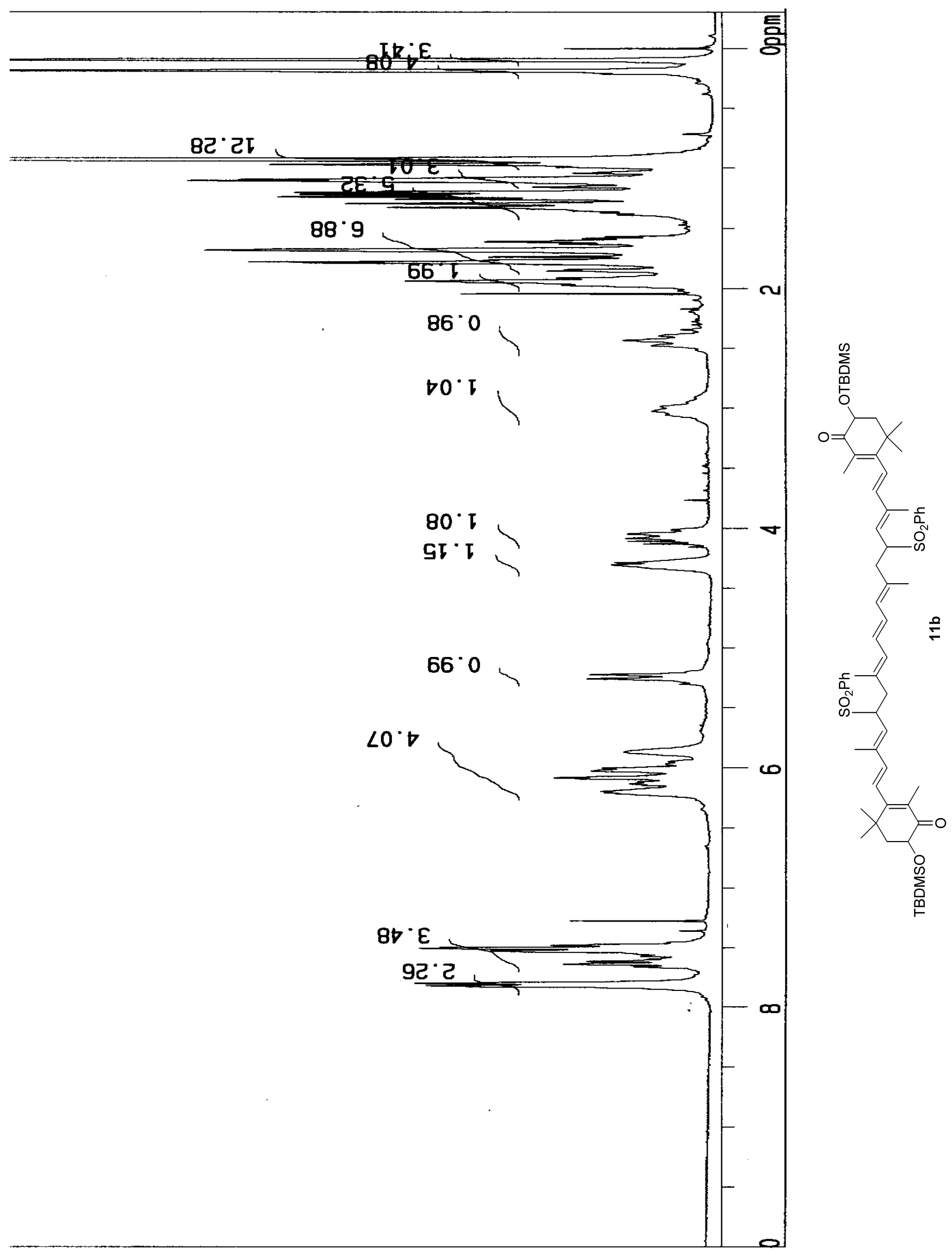


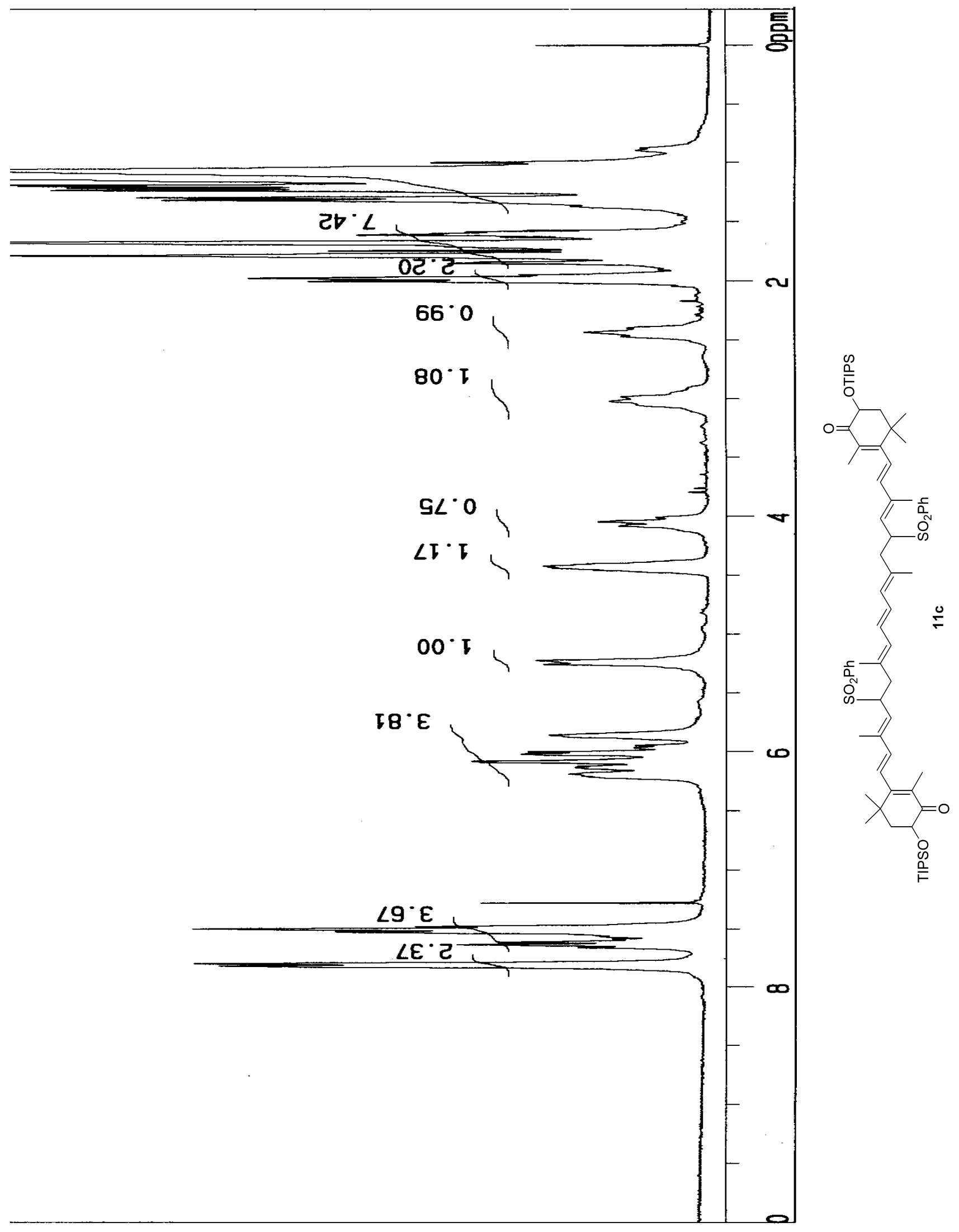




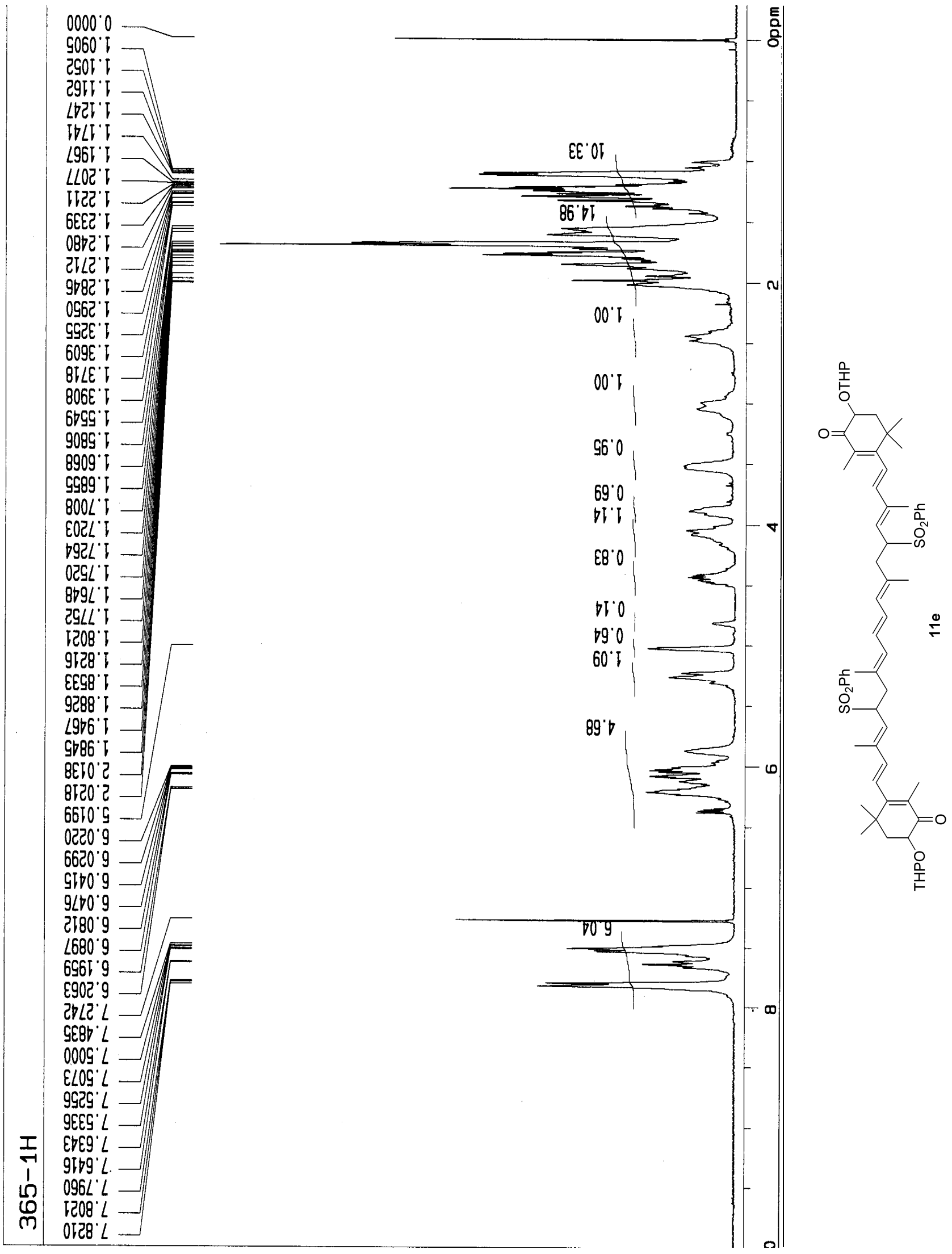

